\title{
Sequencing of the needle transcriptome from Norway spruce (Picea abies Karst L.) reveals lower substitution rates, but similar selective constraints in gymnosperms and angiosperms
}

\author{
Jun Chen ${ }^{1}$, Severin Uebbing ${ }^{1}$, Niclas Gyllenstrand ${ }^{2}$, Ulf Lagercrantz ${ }^{1}$, \\ Martin Lascoux ${ }^{1,3}$ and Thomas Källman ${ }^{1 *}$
}

\begin{abstract}
Background: A detailed knowledge about spatial and temporal gene expression is important for understanding both the function of genes and their evolution. For the vast majority of species, transcriptomes are still largely uncharacterized and even in those where substantial information is available it is often in the form of partially sequenced transcriptomes. With the development of next generation sequencing, a single experiment can now simultaneously identify the transcribed part of a species genome and estimate levels of gene expression.
\end{abstract}

Results: mRNA from actively growing needles of Norway spruce (Picea abies) was sequenced using next generation sequencing technology. In total, close to 70 million fragments with a length of 76 bp were sequenced resulting in 5 Gbp of raw data. A de novo assembly of these reads, together with publicly available expressed sequence tag (EST) data from Norway spruce, was used to create a reference transcriptome. Of the 38,419 PUTs (putative unique transcripts) longer than $150 \mathrm{bp}$ in this reference assembly, 83.5\% show similarity to ESTs from other spruce species and of the remaining PUTs, 3,704 show similarity to protein sequences from other plant species, leaving 4,167 PUTs with limited similarity to currently available plant proteins. By predicting coding frames and comparing not only the Norway spruce PUTs, but also PUTs from the close relatives Picea glauca and Picea sitchensis to both Pinus taeda and Taxus mairei, we obtained estimates of synonymous and non-synonymous divergence among conifer species. In addition, we detected close to 15,000 SNPs of high quality and estimated gene expression differences between samples collected under dark and light conditions.

Conclusions: Our study yielded a large number of single nucleotide polymorphisms as well as estimates of gene expression on transcriptome scale. In agreement with a recent study we find that the synonymous substitution rate per year $\left(0.6 \times 10^{-09}\right.$ and $\left.1.1 \times 10^{-09}\right)$ is an order of magnitude smaller than values reported for angiosperm herbs. However, if one takes generation time into account, most of this difference disappears. The estimates of the dN/dS ratio (non-synonymous over synonymous divergence) reported here are in general much lower than 1 and only a few genes showed a ratio larger than 1.

\footnotetext{
${ }^{*}$ Correspondence: thomas.kallman@ebc.uu.se

1 Department of Ecology and Genetics, EBC, Uppsala University, 75236

Uppsala, Sweden

Full list of author information is available at the end of the article
} 


\section{Background}

A detailed characterization of the transcriptome is not only a prerequisite for genome annotation, but is also crucial to relate gene expression and function to phenotypic variation, to estimate evolutionary rates or to identify genes under selection [1-5]. While the first large-scale EST (Expressed Sequence Tag) projects based on Sanger sequencing had already revealed that transcriptomes are highly diverse and complex, this has over the last decade been corroborated on an unprecedented scale by massive parallel sequencing (MPS) [6-9].

Until recently microarrays, based on either cDNA, or in the case of model organisms, oligonucleotides, were the main tool to assess global patterns of gene expression. These microarray studies provided a first characterization of temporal and spatial gene expression patterns in many different organisms, but they also had obvious limitations since they were dependent on already available sequence information from the organism of interest. Furthermore, at least with cDNA arrays, most transcripts were only represented by the most common splice variant making the gene expression estimates unspecific [10]. The emergence of MPS techniques has profoundly transformed the landscape of both genome and transcriptome sequencing [11]. In the case of transcriptome sequencing it has become possible to obtain both sequence data and estimates of gene expression levels in a single experiment [12]. MPS also allowed for very deep sequencing of transcriptomes revealing in principle all expressed parts of the genome. In mouse (Mus musculus), for example, despite a fully sequenced genome and millions of EST sequences in public databases, MPS of expressed genes from three different tissues revealed several thousand new features of known genes and close to 600 novel gene transcripts [6]. Similar results, with a very large number of previously unknown expressed parts of the genome, were recently obtained in Drosophila melanogaster [13]. In plants, MPS has been used to investigate gene expression and alternative splicing in model organisms. As in animals, large parts of the genome are transcribed (although the majority of transcripts can be assigned to previously identified expressed parts of the genome) and around 30-40\% of the genes have more than one expressed splice variant $[8,9]$. In addition, transcriptome sequencing has been employed in a large array of plant species to create basic genetic resources and to study gene expression dynamics (for example see: [14-20]).

Most efforts have so far focused on angiosperms, less attention being paid to gymnosperms in spite of the availability of several very large EST libraries in the spruce (Picea) and pine (Pinus) genus. By April 2012 there were 313,110 and 186,637 EST sequences deposited at NCBI from Picea glauca and P. sitchensis, respectively. Efforts in the European Picea abies (Norway spruce) have been less intensive and only some 14,000 ESTs had been deposited at NCBI by April 2012. Based on sequence similarity, the data obtained in the North American species have been collapsed into 22,472 putative unique transcripts (PUTs, unigenes) in P. glauca and 19,828 unigenes in P. sitchensis (see Unigene assembly on NCBI). In addition, more focused experimental and bioinformatic efforts have been made to obtain full-length cDNAs (FL-cDNA) in both P. sitchensis and P. glauca and have so far lead to the identification of 27,720 (23,589 annotated as FL-cDNA) unique transcribed genes in P. glauca and around 8,000 validated FL-cDNA have been characterized in P. sitchensis $[21,22]$. These data sets have been very useful for creating microarray assays, detecting polymorphic markers to construct genetic maps and to estimate evolutionary rates [23-26]. Gymnosperms separated from angiosperms some 300 million years ago [27] and they differ in many important aspects. For example, in conifers, the overall recombination seems to be lower than in angiosperms and limited to genes $[28,29]$ and, in contrast to angiosperms where genome duplication has been pervasive, genome duplication is rare in conifers [30]. The generally very large size of conifer genomes is rather a result from massive invasion of transposable elements early in the history of the group [31]. One could therefore expect different mode and tempo of evolution of the transcriptome in these two groups of plants. The gymnosperm group does, compared to the angiosperm group, contain fewer species, but many gymnosperm species have very large distribution ranges and are of great ecological and economical importance [32]. The largest taxon within gymnosperms is the coniferophyta that harbors around 600 species, including many tree species such as pines, spruces and yews [27]. Within these three groups the phylogenetic relationships are not fully known since molecular divergence tends to be very low. For example, within the spruce genera morphological and molecular markers often give conflicting results and multi-locus nuclear DNA data indicates that incomplete lineage sorting is a major challenge for inferring species trees [33]. The spruce lineage separated from the pine lineage around 120-160 millions years ago whereas the Taxus lineage is more distantly related and are believed to have a common ancestor to the pine and spruce lineages between 240 and 300 million years ago [27,34].

In this study we present results from deep sequencing of the $P$. abies needle transcriptome. The data was used to create a reference transcriptome, containing a extensive set of genes expressed in actively growing spruce needles. Combined with available EST from publicly available databases, the size of the characterized part of the transcriptome in $P$. abies now reaches the same order of magnitude as data available from North American spruce species, with thousands of putative full-length genes. We used these data to address the following questions: 
First, to what extent can this type of short sequence data be assembled de novo to full length transcripts? Second, can the short reads be used to identify polymorphic sites? Third, by analyzing mRNA samples from two time points; during day (in the light) and during night (in the dark) can we detect differentially expressed transcripts and if so does the inferred pattern differ compared to angiosperms? Fourth, since recent studies of divergence between conifer species have suggested that there might be major differences in evolutionary rates between conifers and angiosperms [26] we used the assembly together with data from P. glauca, P. sitchensis, Pinus taeda and Taxus mairei to evaluate this statement with a larger and more diverse data set. By comparing not only our, but also two other spruce transcriptome sequences created by traditional sequencing methods, to both Pinus taeda and Taxus mairei we could minimize biases that might exist in any single assembly. Furthermore, since inferring pattern of divergence between species can be biased when closely related species (e.g. incomplete lineage sorting) or highly divergent species (saturation on synonymous sites) are used, the use of species that diverged at two different time points should reduce these problems.

\section{Results}

RNA extracted from needles of a single adult $P$. abies tree growing outside Uppsala, Sweden $\left(59^{\circ} 51^{\prime} 29^{\prime \prime} \mathrm{N}\right.$, $\left.17^{\circ} 48^{\prime} 38^{\prime \prime} \mathrm{E}\right)$ was used to produce 6 different adapterligated cDNA libraries. In total, close to 70 million $76 \mathrm{bp}$ long fragments were sequenced from both ends and close to $50 \%$ of the reads originated from the samples collected in the dark (Table 1). Based on overlap between pairend reads, the obtained library sizes were smaller than the expected 150-300 bp and around 50\% of the reads contained detectable traces of adaptor sequence, which means that the fragment size was smaller than the read length of $76 \mathrm{bp}$. Thus, reads were filtered, not only based on quality, but also on presence of adaptor sequence, reducing the data set to $33,185,901$ fragments that was used in de novo assembly ( $\sim 15$ million where paired end sequences were retained and $\sim 18$ million when only one of the paired reads was retained). For mapping purposes we created a second data set where reads containing adaptor sequence were trimmed rather than removed and retained if they were longer than $30 \mathrm{bp}$ after trimming. This created a data set of 55,417,522 fragments (almost 36 millions sequenced at both ends and 19.5 millions from only one end) of length between 30 and $76 \mathrm{bp}$ (Table 1).

\section{de novo assembly}

Two types of short read de novo assemblers were used. First, both pair-end and single-end reads were assembled using Velvet [35] and second, all reads were treated as single-end reads and assembled using ABySS [36]. The resulting contigs from both assemblers were merged with 8,715 PUTs (putative unique transcripts) assembled from ESTs at plantgdb.org [37] to create a more comprehensive set of PUTs from $P$. abies. The final assembly contained 59,556 PUTs with a N50 and a N90 size of $551 \mathrm{bp}$ and 156 bp, respectively (N50 and N90 are the length of the shortest contigs in a set of contigs sorted in a descending order that includes $50 \%$ and $90 \%$ of the total assembly length). In all downstream analyses we excluded PUTs shorter than $151 \mathrm{bp}$, leaving 38,419 PUTs with a mean length of 471.5 bp. The total assembly length of PUTs longer than $150 \mathrm{bp}$ was just over $18 \mathrm{Mbp}$ and the mean sequence depth of these PUTs as measured by RPKM (reads per kilobase of transcripts per million of mapped reads) was 281 (Figure 1 and Additional file 1).

$55 \%$ of the assembled $P$. abies PUTs showed similarity (blastx E-value $<1 \times 10^{-10}$ ) to plant proteins when translated to protein sequences. Of the 38,419 PUTs 32,082 show a significant hit against either $P$. glauca or $P$. sitchensis and 23,450 of these were similar over more than $90 \%$ of the sequence length of the P. abies PUT. Of the 882 transcripts longer than 1,500 bp only 57 PUTs did not show any similarity to Arabidopsis or other plant

Table 1 Summary of the collected sequence data

\begin{tabular}{|c|c|c|c|c|c|}
\hline \multirow[b]{2}{*}{ RNA source } & \multirow[b]{2}{*}{ Raw PE reads } & \multicolumn{2}{|c|}{ Used in de novo assembly } & \multicolumn{2}{|c|}{ Used in mapping } \\
\hline & & PE reads & SE reads & PE reads & SE reads \\
\hline Light extraction 1 & $28,073,980$ & $5,668,652$ & $3,229,442$ & $14,751,144$ & $3,738,405$ \\
\hline Light extraction 2 & $20,442,438$ & $4,589,450$ & $2,930,651$ & $9,693,554$ & $3,077,721$ \\
\hline Light extraction 3 & $23,554,246$ & $5,183,788$ & $2,929,447$ & $12,575,038$ & $3,217,660$ \\
\hline Dark extraction 1 & $23,885,794$ & $5,004,782$ & $3,054,332$ & $12,080,884$ & $3,359,437$ \\
\hline Dark extraction 2 & $22,157,136$ & $4,929,488$ & $2,929,710$ & $11,349,636$ & $3,084,920$ \\
\hline Dark extraction 3 & $21,643,358$ & $4,981,140$ & $2,933,669$ & $11,307,910$ & $3,060,296$ \\
\hline Total & $139,756,952$ & $30,357,300$ & $18,007,251$ & $71,758,166$ & $19,538,439$ \\
\hline
\end{tabular}

Collected number of sequences from the 6 different sequence libraries. The number of reads used for assembly and mapping divided as pair-end (PE) and single-end (SE) reads retained after cleaning and filtering as described in Material and methods. 


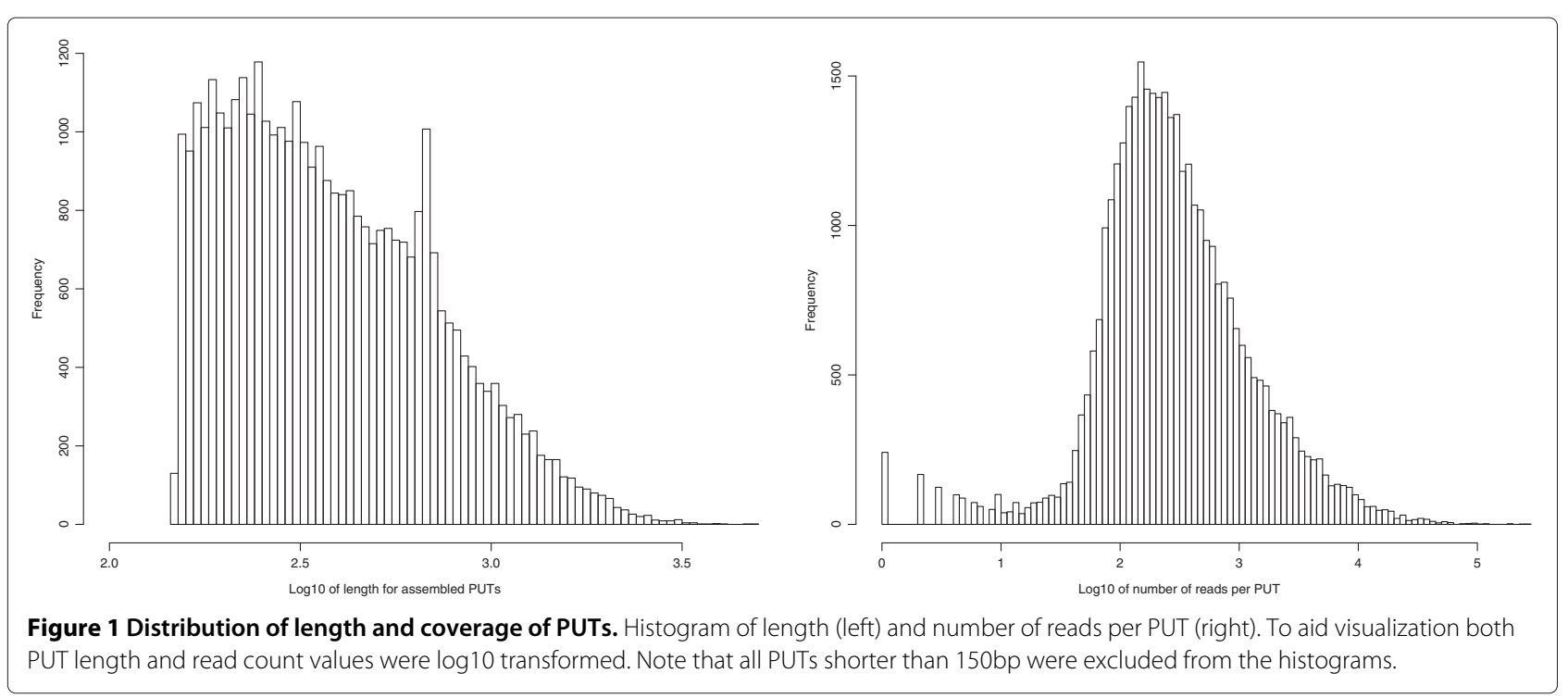

proteins. Taken together this left only 4,167 P. abies PUTs with no or limited similarity to any plant protein sequence or any EST sequence from other spruce species. For complete annotation of PUTs longer than 150 bp against $A$. thaliana, P. glauca, P. sitchensis, plant proteins, PFAM databases and repeat databases see Additional file 1 .

Potential reading frames were identified from all PUTs longer than 150 nucleotides. This led to the identification of 6,194 PUTs that have properties suggesting that they are full-length transcripts including both a start and stop codon and have an open reading frame (ORF) of at least 10 amino acids (Additional file 1). Comparison of the length distribution of both untranslated regions (UTRs) and the ORF length to the validated full-length data set from both P. sitchensis [21] and P. glauca [22] suggests that a fraction of our putative full-length PUTs includes full-length transcripts. However, the length distributions of both the 5'-UTRs, 3'-UTRs and ORFs were enriched for short sequences compared to what was reported from the $P$. sitchensis and $P$. glauca (Figure 2 compared to Figure three in [21]). A direct comparison between a set of potentially orthologous sequences in P. abies and P. glauca shows that there is a large number of PUTs that likely are fulllength as they are equally long in both species, but it is also clear from this comparison that neither of these two data sets are solely composed of full-length transcripts as many transcripts annotated as full-length in either P. glauca or $P$. abies appear to be truncated when compared to each other (Figure 2d). Approximately one fifth of the 6,194 full length $P$. abies transcripts where orthologous sequences were found in P. glauca displayed less than $5 \%$ difference in sequence length and hence likely represents full length transcripts. All PUTs containing coding frames were also annotated by comparing them to the PFAM-A domains. A comparison of the number of different detectable domains in the $P$. abies transcriptome to the $P$. glauca transcriptome is consistent with shorter length of assembled $P$. abies transcriptome. We detected fewer domains of all types in the P. abies assembly, but the distributions from the two species are indeed very similar (Figure 3 ).

\section{Identification of variable sites}

By mapping short reads to the $P$. abies PUT assembly we extracted potential SNPs using samtools and the mpileup function on the mapped reads. The number of SNPs identified was, as expected, largely dependent on the criteria used in the algorithm and as the data is from nonnormalized RNA the commonly used settings for genome sequencing will likely not give satisfactory results. With very stringent criteria, including a cut-off for the observed allele frequency of the minor allele at $25 \%$ (note that as all the data is from one individual we expect segregating alleles to be found at frequency 0.5 unless there are allele specific gene expression), read direction for alternate alleles (DP4) and coverage 9,394 SNPs were found. Less stringent criteria obviously led to a higher number of identified SNPs and if we only filter on quality 20,781 potential SNPs were identified. From published $P$. abies population genetic data set the observed transition to transversion ratio is around 1.3 , which is very similar to the ratio observed with the criteria that includes both quality and observed minor allele frequency above 0.25 (Table 2).

\section{Comparative molecular evolution in conifers}

Previous genetic studies from both nuclear and organellar markers within the Picea group have revealed a fairly shallow phylogeny with partly contrasting results between different marker types [33,38-40]. All these studies have been restricted to either small data sets using at most 10 

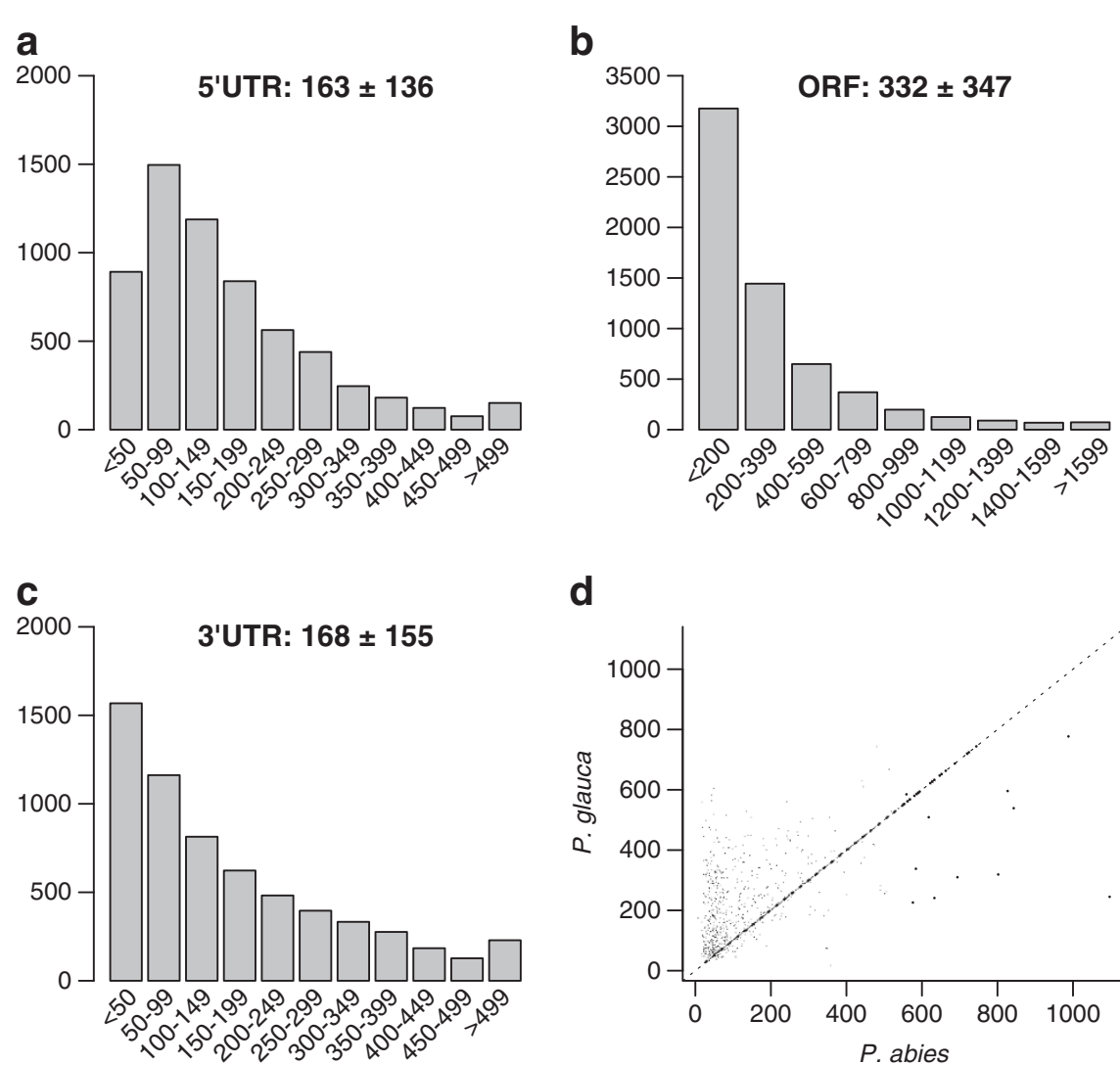

d

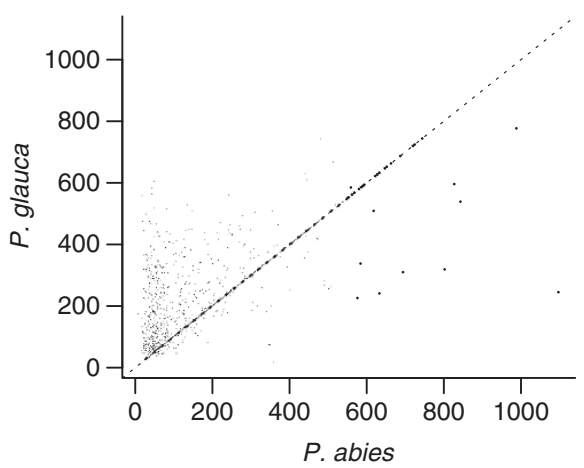

Figure 2 Length distributions of different mRNA features in the $P$. abies PUTs. a) $5^{\prime}$-UTR (untranslated region). b) ORFs (open reading frame). c) $3^{\prime}$-UTR. d) Comparison of putative full-length ORFs in P. abies and P. glauca.

gene fragments or only organelle markers. Here we combine EST data from three spruce species (P. abies, P. glauca and P. sitchensis), a yew (Taxus mairei) and a pine (Pinus taeda) to estimate phylogenetic trees and study the rate of molecular evolution in conifers. In all five species we identified putative coding frames and aligned orthologous sequences from all species. This resulted in a data set of 5,246 aligned ORFs between the three Picea species. This data set was reduced to 1,404 when adding pine and further reduced to 244 when adding yew. Phylogenetic inference on this latter data set confirms the very close relationship between all three spruce species and we cannot with any confidence resolve their phylogeny (Figure 4). Due to this lack of resolution and the large reduction in data sets as more species were added, we restricted the analysis of synonymous and nonsynonymous divergence to pairwise comparisons between one of the three spruce species, on the one hand, and either the pine or the yew, on the other hand. This created 6 pairwise data sets with an average 4,737 ORFs aligned in each comparison (P. abies - P. taeda: 6,977, P. abies - T. mairei: 2,932, P. glauca - P. taeda: 7,183, P. glauca - T. mairei: 3,110, P. sitchensis - P. taeda: 5,773, P. sitchensis - T. mairei: 2,447).
Mean synonymous divergences between the three spruce species and pine and yew were 0.175 (P. abies: 0.172 [95\% confidence interval, $\mathrm{CI}=0.1701,0.1736]$, P. glauca: 0.177 $[\mathrm{CI}=0.1752,0.1783]$, P. sitchensis: $0.176[\mathrm{CI}=0.1743$, $0.1779])$ and 0.598 (P. abies: 0.577 [CI $=0.5711,0.5837]$, P. glauca: $0.605[\mathrm{CI}=0.5979,0.6120]$, P. sitchensis: 0.611 $[\mathrm{CI}=0.6028,0.6190])$, respectively. Assuming a divergence time between pine and spruce of 160 to 120 million years $[26,34]$ and a divergence between spruce and yew of 300 to 240 million years [27] these values correspond to a yearly substitution rate of $0.55 \times 10^{-09}$ to $0.73 \times 10^{-09}$ and $1 \times 10^{-09}$ to $1.24 \times 10^{-09}$, respectively. The ratio of non-synonymous to synonymous divergence between the spruce species and pine and yew show very few genes with ratios larger than one, irrespective if the analysis were performed on only full-length ORFs or on all identified ORFs (Figure 5).

\section{Differentially expressed genes}

Since sequence data were collected from three technical replicates sampled in the dark during night and three replicates sampled during daytime in normal daylight conditions, we set out to detect differentially expressed 


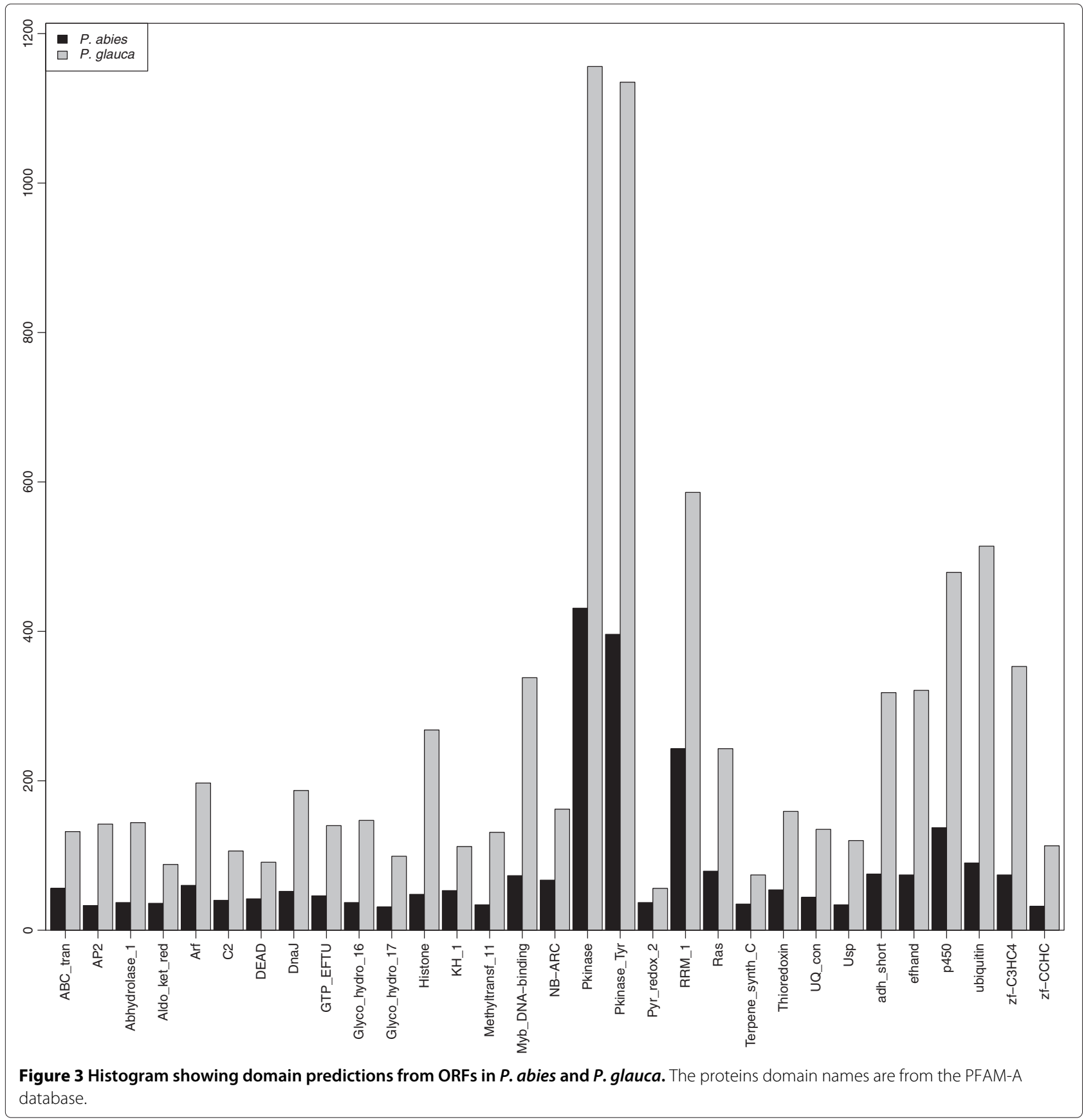

genes. The correlations of estimated levels of gene expression based on just counting the number of pair-end hits against any given reference gene were in general very high especially for the dark samples (data not shown). 2,076 transcripts were detected as significantly differentially expressed between the two sampling points using BaySeq and a cut-off posterior probability at 0.5 , but among these transcripts only few showed a large fold change (Figure 6, Additional file 1). We did not observe any significant correlation between either expression level or differential expression and selective constraint as measured by $\mathrm{dN} / \mathrm{dS}$.

\section{Discussion}

In spite of the remaining challenges associated to the assembly of short reads to full-length transcripts [41] RNA-seq has over the last years evolved into the de-facto standard for transcriptome analysis even in non-model species $[11,42]$. In the present study we evaluated the quality of our assembly using a set of different criteria largely 
Table 2 Putative single nucleotide polymorphisms at different quality criteria

\begin{tabular}{|c|c|c|c|c|}
\hline Filter & SNP & Transitions & Transversions & Ratio \\
\hline Sanger Qual $>=60$ & 20,781 & 11,389 & 9,392 & 1.21 \\
\hline Sanger Qual $>=60$ and AF $[0.25,0.75]$ & 14,745 & 8,372 & 6,372 & 1.31 \\
\hline Sanger Qual $>=60$ and AF $[0.25,0.75]$ & 9,394 & 5,622 & 3,770 & 1.49 \\
\hline and Depth $>=20$, DP4 $>=10$ & & & & \\
\hline
\end{tabular}

The effect of using different quality criteria for identifying SNPs. Sanger Qual = Sanger sequence quality, AF= Allele frequency, Depth= sequence depth at the base DP4=Depth of the two variants according to the read direction. For details see the samtools specifications.

relying on comparisons to other plant species. First, we compared basic summary statistics to data from other conifer species, where the transcriptome was obtained through Sanger sequencing. Second, we further characterized the assembly by assessing properties like transcriptome annotation and similarity at the protein level to available plant protein sequences. P. glauca, which has one of the best characterized transcriptome among conifer species, has a transcriptome assembly length of 30.15 $\mathrm{Mbp}$, i.e. $12 \mathrm{Mbp}$ longer than the the $P$. abies assembly presented here. In addition, the length of individual
PUTs is on average shorter in the $P$. abies assembly than in both P. glauca and P. taeda (Figure 1, Figure 2, http:// www.plantgdb.org). This indicates that the $P$. abies transcriptome assembly only comprises a fraction of the total transcriptome and that a large proportion of the assembled PUTs corresponds to partial transcripts. There are a number of possible reasons for this difference in assembly length despite of the large amount of short reads collected. First, the assembly of short reads from transcriptome sequence is still far from optimal [41], something that likely is elevated in this data set as the mean fragment

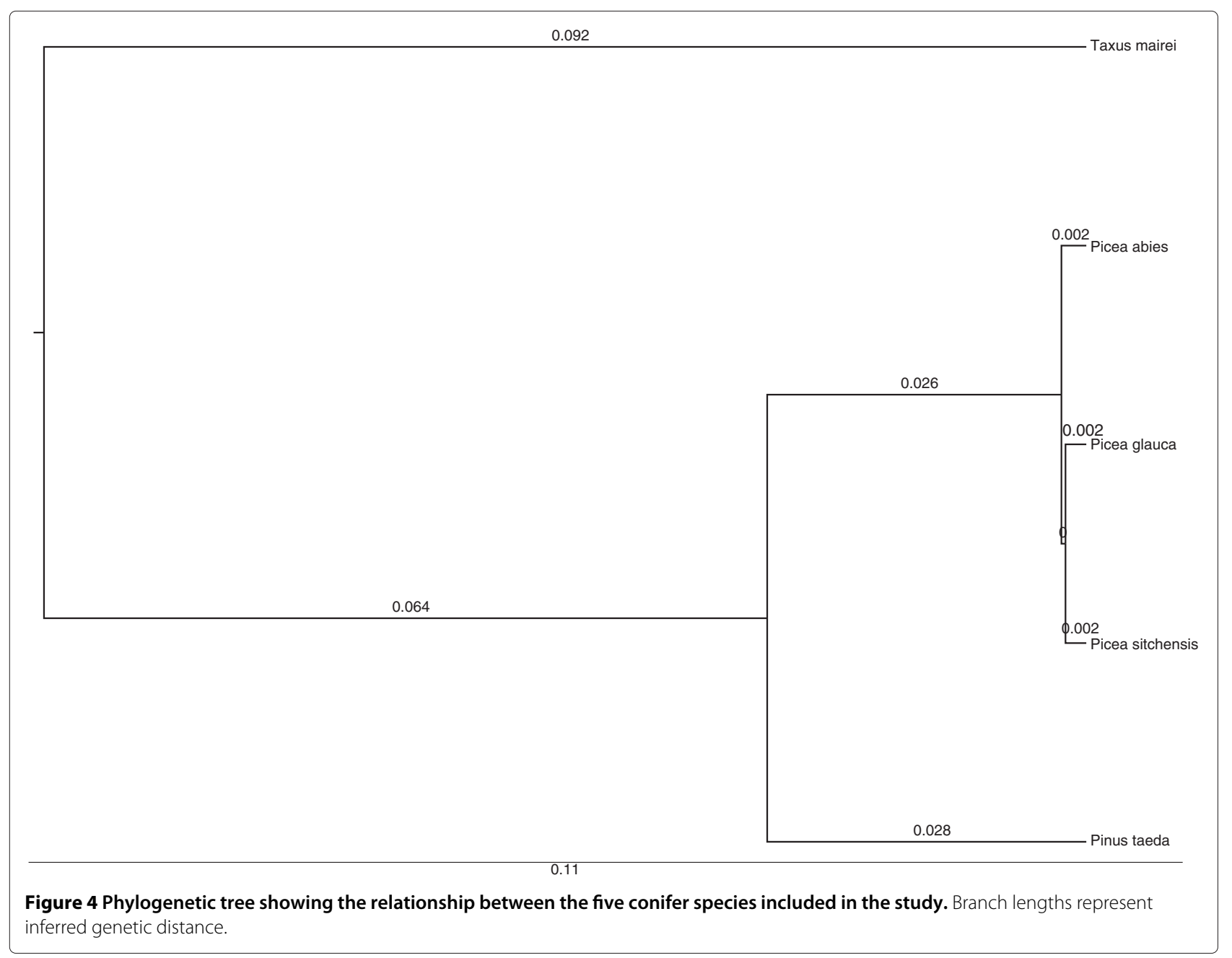




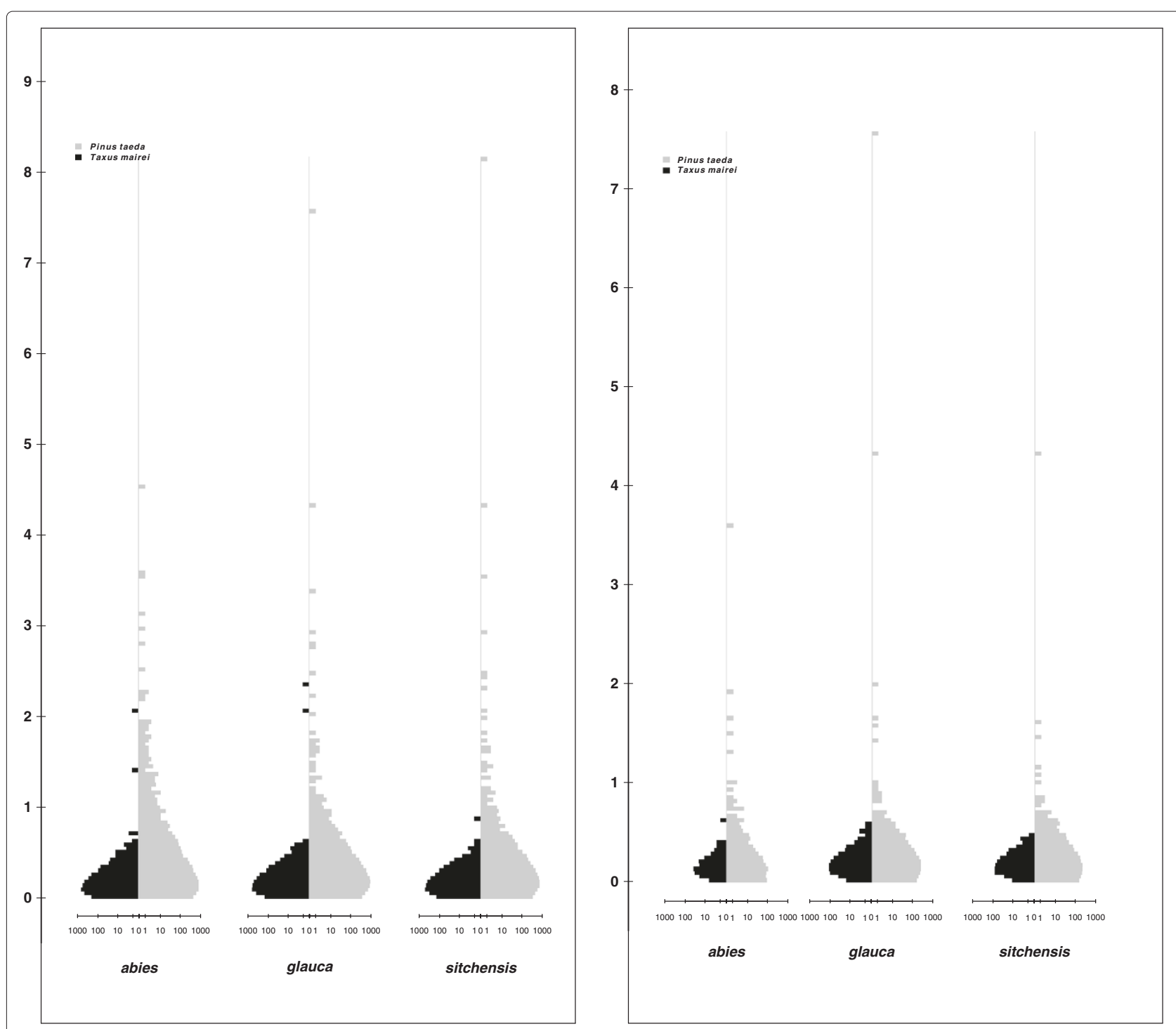

Figure 5 Histograms showing the distribution of $\mathbf{d N} / \mathrm{dS}$ values from pairwise comparisons of species. The left plot shows pattern for all potential orthologous sequences in the data set whereas the right plot shows the pattern when restricting the data sets to only putative full-length ORFS.

length in the sequence library was shorter than the pairend distance. Second, non-normalized mRNA was used, which means that the read depth of lowly expressed genes is likely insufficient for assembly. Third, the short read libraries used here obviously will capture only a restricted part of the total transcriptome as they are based on a single tissue and only two sampling time points. All this is in contrast to the other mentioned conifer species EST assemblies that are, at least partially, composed of normalized mRNA libraries and also include mRNA extracted from several tissues including root, which in general have divergent expression patterns $[21,22,43,44]$.

In total over 34,000 transcripts showed significant hits when compared to other spruce species, either at the protein level to plant proteins or at the nucleotide level, leaving only 4,167 PUTs without any annotation. This set of PUTs with low or no similarity to other species could represent erroneously assembled PUTs, but since estimates from the P. glauca transcriptome suggest that as much as $11.6 \mathrm{Mbp}$ of the transcriptome is still missing [22], they could also represent previously undiscovered spruce transcripts. In theory they could also be transcripts specific to $P$. abies, but this seems less likely as both this data and previous studies have shown that spruce species are very closely related and even share many polymorphic sites $[33,39]$. Still, genome size between different spruce species is variable $(15.8-20.2 \mathrm{pg} / 1 \mathrm{C}$ [45]), which could imply significant differences in gene content 


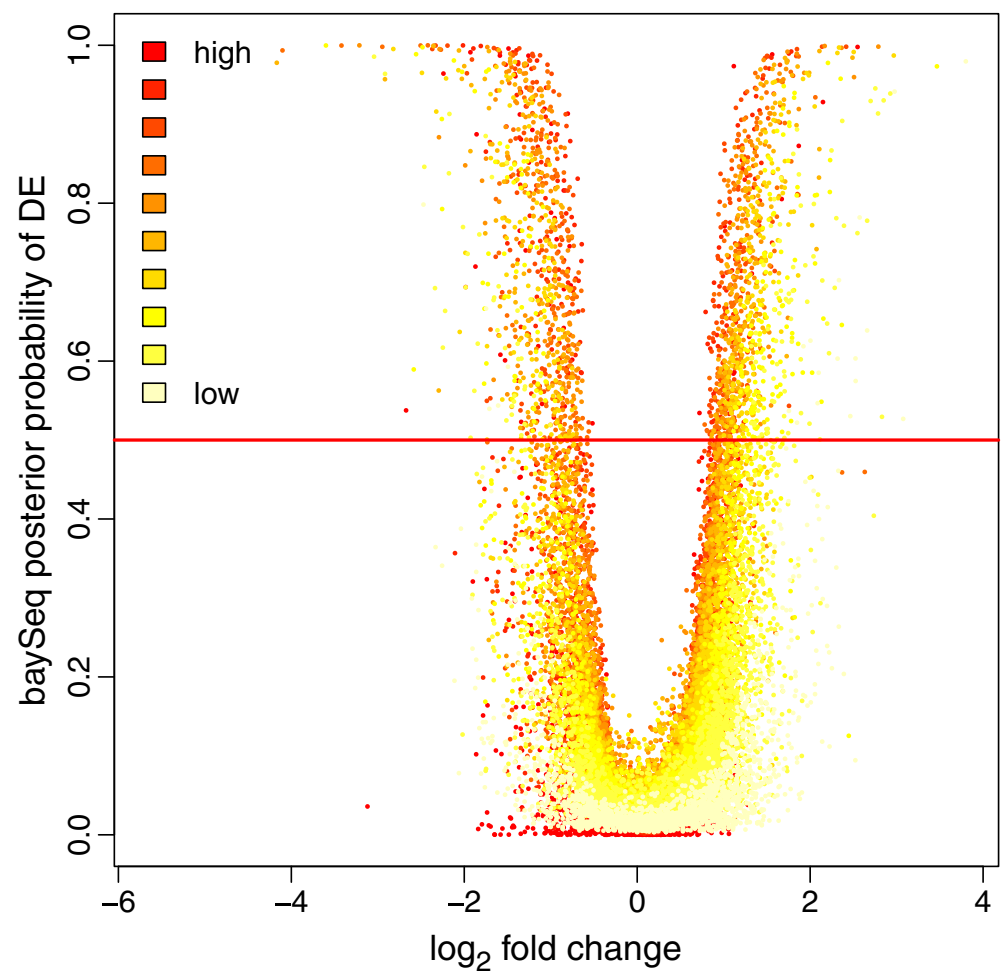

Figure 6 Volcano plot of gene expression pattern. The $y$-axis represent the posterior probability of differential expression and the fold change in $\log 2$ on the $x$-axis. Dots are colored to represent estimated expression level. The red line indicate a cut-off value of 0.5 for the posterior probability of differential expression.

between them. Finally, and perhaps most likely, they may represent assemblies containing mainly non-coding RNA and partial transcripts with mostly UTR sequence that, in general, show lower degree of conservation between species. In summary, even if the $P$. abies transcriptome contains a large fraction of partial transcripts and is far from complete, the assembly presented here is of a high enough quality and size to be informative on the general properties of the transcriptome.

In fact, ORF predictions identified 6,194 PUTs from $P$. abies with properties that suggest that they are fulllength transcripts. A direct comparison of these PUTs towards full-length transcripts from P. glauca indicate that neither ours nor the P. glauca PUT assembly contains a comprehensive set of full-length transcripts and that despite millions of ESTs from spruce species there is still room for efforts aiming at capturing and sequencing fulllength transcripts, such as oligo-capping, something that is of special importance in relation to upcoming genome annotations [46].

In many of the PUTs with high enough sequence coverage we could extract potentially variable sites, but since sequencing technologies are evolving at a rapid pace, the understanding of the error profiles for a given technique is still sparse making this process nontrivial and prone to false positives. This process is, in the present case, further complicated by the fact that the majority of tools developed to extract SNPs assumes that genome data is available and that sequence depth is even for the underlying genotypes, meaning that one expects around half the reads from each allele at heterozygous sites. In addition, many tools make use of uneven coverage as a tool to detect copy number variants or duplicated regions making standard methods to discover SNPs difficult to use as the uneven coverage and allele specific gene expression will cause deviations from these expectations. By merging population genetics sequence data sets from both unpublished and published projects in $P$. abies, where the data have been collected with Sanger sequencing $[33,47]$ we compared the ratio of transitions to transversions to the ratio obtained here under different cut-offs. Adding both quality and coverage of the alternate alleles as criteria yielded almost identical transition to transversion ratio as the Sanger data, suggesting that this criteria might be suitable for this data set (Table 2). Hence, from a single individual study we have identified close to 15 thousand SNPs, but because all of these stem from only one individual a fraction of them will be either singletons or 
low frequency variants within the species. Even so these results suggests that mRNA sequencing can be of great use for the identification of population genetic markers. However, recent discussions have identified several issues in identification of variable sites from cDNA something that calls for caution and further validation before using SNPs from cDNA as a source for identification of genetic markers [48-50].

The observed mean synonymous divergence of 0.175 between spruce and pine obtained here, is similar to the 0.22 reported as a mean over a phylogenetic tree of four conifer species [3] and the 0.19 reported from a pairwise comparison of P. sitchensis and P. taeda [26]. In the comparisons to yew we obtained a mean silent divergence around 0.6. These divergence estimates translate into average synonymous substitution rates of $0.6 \times 10^{-09}$ and $1.1 \times 10^{-09}$, values that are also in line with previous estimates of $0.7-1.31 \times 10^{-09}$ [51]. As previously noted this yearly synonymous substitution rate is much lower than estimates of substitution rate from annual angiosperm species. Data from numerous studies have obtained similar patterns and there is now substantial evidence supporting a lower annual substitution rate in gymnosperms compared to many annual angiosperms $[3,26,34,51]$. Since lower synonymous substitution rates per year have also been observed in angiosperms trees/shrubs compared to herbs [52,53] one of the main causes seem simply to be that gymnosperms are trees with a long generation times and if substitution rates are calculated per generation rather than per year, the estimates between perennial gymnosperms presented here are similar to estimates from annual angiosperms. The mechanisms underlying the slower substitution rates in perennials than in annuals remain unclear $[52,54]$ and we will not speculate on its causes here. Interestingly though, available data also suggest that polymorphism level is lower in trees, and in particular in conifers, than in herbs (Additional file 2). This apparent effect of generation time on nucleotide diversity could reflect the fact that organisms with long generation time and low substitution rate will require longer period of time to recover from past decline in effective population sizes. Also, for a given number of generations they will span much longer periods of time than annuals and thereby will be more likely to experience environmental changes causing variation in population size.

The ratio of non-synonymous to synonymous divergence at full length ORFs between spruce species and either pine or yew reported here, $0.236(\mathrm{CI}=0.2272$, $0.2432)$ and $0.167(\mathrm{CI}=0.1614,0.1717)$, respectively, are higher than estimates from previous analyses in conifer species [3], where branch specific estimates were used (with values of $0.12,0.14$ and 0.15 for the internal branch, the branch leading to P. glauca and the branch leading to $P$. menziesii, respectively), but lower than the pairwise estimate between P. taeda and P. sitchensis (0.314 (95\% $\mathrm{CI}=0.299,0.329)$ ) [26]. The deviation from the former study is likely due to the fact that they included only sequences present in all four conifer species used in their study thereby limiting severely the number of sequences (138) and likely enriching the data set for conserved sequences. The difference with the latter study is harder to explain, but stems mainly from differences in the way coding frames and orthology were defined. In Buschiazzo et al. [26] 100 comparisons revealed a dN/dS ratio higher than one, and most of these estimates are based on short alignments where both the number of synonymous sites and changes were small. This is in stark contrast to our results with very few values larger than one and a weaker correlation between $\mathrm{dN} / \mathrm{dS}$ ratios and alignment length (Figure 5, Additional file 3). Buschiazzo et al. [26] also estimated dN/dS between Arabidopsis thaliana and Populus trichocarpa and obtained a value of 0.0924. They therefore concluded that $\mathrm{dN} / \mathrm{dS}$ was higher in conifers than in angiosperms. While our results for the conifer data are within the same order of magnitude, it is worth noting that the estimate they obtained between $A$. thaliana and $P$. trichocarpa tends to be much lower than previous estimates from angiosperms. For example, in estimates comparing $A$. thaliana and $A$. lyrata the mean over more than 5000 genes was 0.203 and within the poplar lineage $P$. trichocarpa and $P$. tremula the mean over almost 600 genes was $0.175[54,55]$. It is also worth noting that the observed synonymous divergence between pine and spruce are more similar to the values obtained within the Arabidopsis and Populus lineages and since synonymous divergence seem to have a strong impact in estimates of $\mathrm{dN} / \mathrm{dS}$ [56] comparing ratios over different groups of species should only be done when the observed synonymous divergence is similar. The latter, together with other uncertainty factors discussed above, suggests that caution is warranted before concluding that there are biologically meaningful differences in selective constraints between angiosperms and gymnosperms.

Even when the correct orthologous sequences have been aligned, individual estimates of $\mathrm{dN} / \mathrm{dS}$ values should not be directly used to infer positive selection as recent work have highlighted several problems in using $\mathrm{dN} / \mathrm{dS}$ larger than one as indicative of positive selection [56-58]. For example, the vast majority of human genes with $\mathrm{dN} / \mathrm{dS}$ ratio larger than one is due to low dS values rather than high $\mathrm{dN}$ values. Hence, the classic interpretation of high $\mathrm{dN} / \mathrm{dS}$ ratios is questionable as relatively low $\mathrm{dS}$ values will have the same effect on the ratio as high $\mathrm{dN}$ values. Furthermore, species pairs that are too closely related will tend to overestimate $\mathrm{dN} / \mathrm{dS}$.

In model organisms large scale studies of gene expression have identified numerous properties of genes and proteins that significantly correlate with selective 
constraints. Here we investigated whether level of gene expression in needles correlated with selective constraints, but found no significant pattern. In animals, fungi and plants a correlation between expression and selective constraint, such that highly expressed genes tend to have lower $\mathrm{dN} / \mathrm{dS}$, has been reported $[54,59,60]$. In multicellular organisms the correlation between $\mathrm{dN} / \mathrm{dS}$ and expression breadth (number of tissues where genes are expressed) is often stronger than with the actual level of gene expression $[54,55,61]$. The lack of correlation in spruce could hence be due to the fact that we only measured gene expression in needles and have no data on expression breadth. Also, since our transcriptome reference sequence is largely created from short reads from needle RNA our reference is biased and contains a specific set of genes that do not fully represent the complete transcriptome; e.g our approach has limited information from genes lowly expressed in needles. The lack of correlation reported here should therefore be taken with a grain of salt as it might simply stem from lack of data rather than from an actual lack of correlation. Analysis of level of gene expression in samples collected in the dark compared to samples collected in the light revealed only a small set of genes showing at least a 2-fold difference in gene expression. Angiosperms studies suggest that around $20 \%$ of the transcriptome is differentially expressed between light and dark treatments, even though the number of genes varies depending on species, tissue and actual treatment [62-64]. The pattern observed in our data is different and might suggest that the diurnally expressed genes in gymnosperm trees could be fewer than in angiosperms. This is consistent with earlier studies, reporting a lack of clear diurnal expression pattern at key photosynthesis genes in gymnosperm species $[65,66]$. To be noted here is that all expression estimates put forward here stems from a single individual and is hence not suitable for making strong statements at the species level.

\section{Conclusions}

Our study highlights the versatility of next generation sequencing technology in generation of full-length expressed genes, identification of polymorphic sites and estimation of gene expression levels. The available data from Norway spruce have with this study been leveraged with available data from North American spruce species. This allowed the comparison of sequence evolution in this group of plants for thousands of full-length genes. By comparing our $P$. abies assembly to other gymnosperm species our analysis suggests that evolutionary constraints might not, as some previous work have suggested, be very dissimilar between gymnosperm and angiosperm species. More importantly, our analysis does, together with the analysis of Buschiazzo et al. [26], highlights some of the problems associated with inference of selection from
$\mathrm{dN} / \mathrm{dS}$ in systems without annotated genome data. Even with a reference genome it is often difficult to unambiguously identify correct ORFs and orthologous sequences and one should consequently refrain from strong statements about patterns of divergence and selection in systems without high quality annotation data. However, as the collection of transcriptome sequence data is getting more streamlined and standardized (for example [67]) it will soon be possible to sample both more individuals and species and thereby facilitates more accurate characterization of selective constraints. The addition of more species will also make it feasible to pinpoint on which branches of evolutionary trees selection has been acting [5]. Furthermore, the recent in-depth analysis of factors contributing to protein evolution in model plants $[54,61]$ will be within reach even in species without a reference genome and will hopefully reveal if there indeed is a difference in selective constraints between angiosperms and gymnosperms.

\section{Methods}

\section{Plant material and RNA extraction}

Needles from a single adult individual of Norway spruce growing naturally outside Uppsala, Sweden were collected at two different time points, at $1 \mathrm{pm}$ during day light conditions and at 1 am the following night in the dark (20090527). The collected needles were actively growing and at stage 5 to 6 according to Krutzsch scale [68]. Extraction of total RNA was done from three replicates for each time point using $2.0 \mathrm{~g}$ of frozen needle tissue with the method described by Kolosova [69]. For all six samples around $400 \mu \mathrm{g}$ of total RNA was obtained of which close to $1 \%$ were retained as mRNA after two rounds of oligo-dT selection using the MicroPoly(A)Purist ${ }^{T M}$ (AppliedBiosystems).

\section{Preparation of cDNA libraries for cluster sequencing}

A high concentration of random hexamer in relation to mRNA was used to synthesize cDNA with a short average fragment size using Invitrogen Supercript III FirstStrand Synthesis kit. Following second strand synthesis, end-repairing of fragments, adaptors for short read pairend sequencing supplied by Illumina corporation were attached to newly synthesized cDNA. The obtained cDNA library had an average size of 150-300 bp (the obtained length was actually shorter; see Results) and were used in ligation of adaptors for cluster sequencing. Enrichment of fragments with two attached adaptors was done using $2 \mu \mathrm{l}$ of purified ligation reaction in a $50 \mu \mathrm{l}$ PCR for 18 cycles. A final purification of the fragment libraries was done by separating the samples on agarose and performing a gel extraction with GE health cares Illustra kit. Before sequencing, the fragment sizes and concentrations were evaluated using Bioanalyzer 2100 and equimolar 
quantities of the library were used for sequencing on Illumina cluster station. A detailed description of the library preparation method can be found in [70].

\section{Sequence filtering and bioinformatic analysis}

The raw sequence data were obtained using the Illumina python pipeline v. 1.3. The obtained sequences were then compared to a number of different potential sources of contamination, including the human transcriptome, rRNA data sets, chloroplast and mitochondrial genomes of spruce, and the genome of $E$. coli. In total, less than $5 \%$ of the reads mapped against these sequence libraries. Following this we created two sets of filtered sequence libraries, one for de novo assembly and one for mapping purposes. For both libraries we retained only high quality reads (quality $>35$ for more than $90 \%$ of the read). For the de novo libraries we removed all reads showing traces of adaptor sequences, whereas for the mapping libraries we trimmed reads showing similarity to adaptor sequences and retained the read if it was longer than $30 \mathrm{bp}$. For the de novo library we also filtered artifact reads and removed any read with homopolymeric regions longer than $20 \mathrm{bp}$. We further removed the first 10 and last $11 \mathrm{bp}$ of the reads, since the start of reads showed a different nucleotide composition compared to the rest of the sequence and the end of reads showed a decrease in quality (data not shown). This led to a de novo assembly with 33,185,901 reads of 55 bp length $(\sim 15$ million sequenced in both ends and $\sim 18$ million from only one end), whereas mapping was done with 55,417,522 fragments (almost 36 millions sequenced in both ends and 19.5 million from only one end) of length between 30 and $76 \mathrm{bp}$.

Two different types of analyses of the read data were performed. First, de novo assembly of the obtained short read data was performed using a combination of two different assemblers followed by a step where putative unique contigs (PUTs) were created. We used two different programs and approaches for the de novo assembly since different assemblers often outcompete each other at different tasks [71]. The pair-end and single-end reads from all libraries were assembled using the short read assembler Velvet [35] using a combination of different $\mathrm{k}$-mer lengths and expected coverages. In parallel, individual libraries were assembled as single end reads, treating forward and reverse reads as independent, using the program ABySS [36]. In total, this yielded one assembly from Velvet and 12 assemblies from ABySS. These assemblies and available EST data from Norway spruce were then merged into putative unique transcripts (PUTs) with the TGICL pipeline [72] if the identity of sequences were $95 \%$ or higher and showed at least 40 bp overlap. Second, we mapped the original short reads to the newly created PUTs which was used as a reference. Mapping was done with BWA [73] aligning pairs of reads and allowing maximum insert size to $500 \mathrm{bp}$ and keeping only reads with mapping quality higher than 20 .

In order to identify differentially expressed genes we used the BaySeq package [74], which is a part of the Bioconductor program suite. BaySeq implements an empirical Bayesian approach to estimate posterior probabilities of gene expression between treatments. The analysis was made on count data obtained by mapping our short read library to the created $P$. abies PUTs.

Finally, single nucleotide polymorphisms were detected using the mpileup function in SAMtools [75] on the short read alignment file against $P$. abies created with BWA. Since there is very limited knowledge about allele specific expression patterns we chose to only present SNPs with very high quality and having a estimated minor allele frequency in our short reads of at least 0.25 .

All raw sequences is available at the NCBI SRA at the accession number SRA053572. A fasta file with with all PUTs as well as SNP information and PAML tables are available at DRYAD under DOI 10.5061/dryad.ds2gp.

\section{Analysis of selective constraints}

The transcriptome sequence data used for inference of phylogenetic relationship and calculation of synonymous and non-synonymous divergence were collected from the following sources: EST sequences available at NCBI Genbank, PUTs assembled at http://plantgdb.org [37], our own sequence assembly and a short read assembly of Taxus mairei [19]. Sequences from P. sitchensis were downloaded from Genbank with the key word 'FLI' and merged with Putative Unique Transcripts (PUTs) from plantgdb. P. glauca sequences came from the 27,720 transcripts from the $P$. glauca gene catalogue [22] and merged with PUT sequences from plantgdb. Sequences of Pinus taeda consisted of PUTs from plantgdb. The phylogenetic relationship between species was inferred using the program BEST [76] largely following the procedure described in [33], but running two chains for 10 million steps.

We used the program getorf from the Emboss software suite to get all possible ORF predictions falling between two stop codons (hereafter: stop1 for 5' stop codon and stop2 for 3' stop codon) [77]. Sequences were based on predictions assigned to six different categories: 1) presence of a start codon between stop1 and stop2; 2) only a start and stop 2 codon present; 3) only a start and stop1 codon present; 4) only a stop2 codon present; 5) only a start and no stop codon present and finally 6) no start or stop codon present. Based on these categories we only considered category 1 as putative fulllength ORFs and all others as partial ORFs (even though sequences assigned to category 2 could be full-length transcripts). We only kept ORFs after the start codon if stop2 was found and before stop1 if it was found. If the two longest ORF predictions on a given sequence 
had a length difference within 5 amino acids we kept the one assigned either as category 1 or 2 as the most likely ORF. For all other sequences we used the longest ORF in downstream analysis.

To evaluate our ORF prediction method, we tested our method on TAIR10 cDNA representative gene model (released on 2011-10-03) and compared our predictions to the peptide sequence database that consisted of 27,416 protein sequences. A total of 27,384 ORF predictions were found with good blastn quality against the protein database (median e-value $=0$, median score $=717$, median percentage of identity $=100 \%$ and both median query coverage and hit coverage $=100 \%$ ). Among those, 26,867 ORFs have the best blast hit with their own proteins, which means that our procedure predicted the correct ORF in more than $98 \%$ of times.

Pairwise Reciprocal Blast hit approach was used to infer putative 1:1 orthologues between Picea species (P. abies, $P$. glauca and P. sitchensis) and Pinus taeda and Taxus mairei using blastn with threshold combination of e-value $=$ $1 \times 10^{-05}$, score $=200$ and percentage of identity $=90 \%$ (for Picea vs Pinus) and 80\% (for Picea vs Taxus). Peptides of predicted ORFs from both species were aligned using the program kalign [78]. To avoid alignment with too large divergence due to problematic ORF predictions from either species, we set a threshold for the percentage of identical amino acids to $50 \%$ and restricted the hits to contain less than 5 and 7 consecutive mismatches in Picea vs Pinus and Picea vs Taxus, respectively. This procedure resulted in a data set of putative orthologues between the different Picea species and either Pinus taeda or Taxus with all loci having E-values lower than $3 \times 10^{-50}$ and $1 \times$ $10^{-50}$, respectively. Nucleotide alignment was performed based on the protein alignments using revtrans [79]. Finally, dN/dS ratios between Picea and Pinus, and Picea and Taxus were estimated using codeml from PAML v. 4.4 [80], with settings seqtype $=1$, runmode $=-2$, CodonFreq $=2$ and transition-transversion ratio estimated from the data. Confidence intervals for the divergence estimates were obtained by bootstrap functions in $\mathrm{R}$.

\section{Additional files}

Additional file 1: Annotation file. Annotation and level of gene expression for the Picea abies PUTs. Details on the meaning of the different columns can be found on Sheet 1 .

Additional file 2: Boxplot showing silent diversity estimates from different group of plant species.

Additional file 3: ORF length and dN/dS. Comparison of the relationship between dN/dS and alignment length with Buschiazzo [26] data on the left and data from our study on the right.

Competing interests

The authors declare no competing interests.

\section{Authors' contributions}

TK, NG and UL designed the experiment. TK collected the needle material and did the wet-lab procedures. SU, JC and TK performed the bioinformatic analysis of the data. TK, JC, UL and ML drafted the manuscript. All authors contributed to and approved the final version of the manuscript.

\section{Acknowledgements}

This work was supported by the European Community's Sixth Framework Programme, under the Network of Excellence Evoltree; by the Seventh Framework Programme (FP7/20072013), under grant agreement 211868 (Project Noveltree), the Eranet Biodiversa LINKTREE project and the Nilsson-Ehle foundation. We also want to acknowledge the Uppsala SNP \& SEQ platform that performed the Illumina sequencing. We are thankful to Samuel Fox for releasing early versions of their library preparation method and for kindly answering our questions concerning the method. We also want to thank Da Cheng Hao and co-authors for sharing their Taxus mairei PUT assembly with us. The final version of the article was greatly improved due to comments from two anonymous reviewers.

\section{Author details}

${ }^{1}$ Department of Ecology and Genetics, EBC, Uppsala University, 75236 Uppsala, Sweden. ${ }^{2}$ Department of Plant Biology and Forest Genetics, Swedish University of Agricultural Sciences, Uppsala, P.O. Box 7080, SE-750 07 Uppsala, Sweden. ${ }^{3}$ Laboratory of Evolutionary Genomics, CAS-MPG Partner Institute for Computational Biology, Chinese Academy of Sciences, Shanghai, China.

Received: 27 April 2012 Accepted: 25 October 2012

Published: 2 November 2012

References

1. Clark AG, Glanowski S, Nielsen R, Thomas PD, Kejariwal A, Todd Ma, Tanenbaum DM, Civello D, Lu F, Murphy B, Ferriera S, Wang G, Zheng X, White TJ, Sninsky JJ, Adams MD, Cargill M: Inferring nonneutral evolution from human-chimp-mouse orthologous gene trios. Science 2003, 302(5652):1960-1963.

2. Jørgensen FG, Hobolth A, Hornshøj H, Bendixen C, Fredholm M, Schierup $\mathrm{MH}$ : Comparative analysis of protein coding sequences from human, mouse and the domesticated pig. BMC bio/ 2005, 3:2.

3. Palmé $A E$, Wright $M$, Savolainen $O$ : Patterns of divergence among conifer ESTs and polymorphism in Pinus sylvestris identify putative selective sweeps. Mol Biol Evol 2008, 25(12):2567-2577.

4. Künstner A, Wolf JBW, Backström N, Whitney O, Balakrishnan CN, Day L, Edwards SV, Janes DE, Schlinger Ba, Wilson RK, Jarvis ED, Warren WC, Ellegren $\mathrm{H}$ : Comparative genomics based on massive parallel transcriptome sequencing reveals patterns of substitution and selection across 10 bird species. Mol Ecology 2010, 19(Suppl 1):266-276.

5. Lee EK, Cibrian-Jaramillo A, Kolokotronis SO, Katari MS, Stamatakis A, Ott M, Chiu JC, Little DP, Stevenson DW, McCombie WR, Martienssen RA Coruzzi G, DeSalle R: A functional phylogenomic view of the seed plants. PLoS Genet 2011, 7(12):e1002411.

6. Mortazavi A, Williams BA, McCue K, Schaeffer L, Wold B: Mapping and quantifying mammalian transcriptomes by RNA-Seq. Nat Methods 2008, 5(7):621-628.

7. Pan Q, Shai O, Lee LJ, Frey BJ, Blencowe BJ: Deep surveying of alternative splicing complexity in the human transcriptome by high-throughput sequencing. Nat genet 2008, 40(12):1413-1435.

8. Filichkin Sa, Priest HD, Givan Sa, Shen R, Bryant DW, Fox SE, Wong WK Mockler TC: Genome-wide mapping of alternative splicing in Arabidopsis thaliana. Genome Res 2010, 20:45-58.

9. Zhang G, Guo G, Hu X, Zhang Y, Li Q, Li R, Zhuang R, Lu Z, He Z, Fang X, Chen L, Tian W, Tao Y, Kristiansen K, Zhang X, Li S, Yang H, Wang J, Wang $\mathrm{J}$ : Deep RNA sequencing at single base-pair resolution reveals high complexity of the rice transcriptome. Genome Res 2010, 20(5):646-654

10. Draghici S, Khatri P, Eklund AC, Szallasi Z: Reliability and reproducibility issues in DNA microarray measurements. Trends Genet : TIG 2006, 22(2):101-109.

11. Hamilton JP, Robin Buell C: Advances in plant genome sequencing Plant J 2012, 70:177-190.

12. Wilhelm BT, Landry JR: RNA-Seq-quantitative measurement of expression through massively parallel RNA-sequencing. Methods 2009, 48(3):249-257. 
13. Graveley BR, Brooks AN, Carlson JW, Duff MO, Landolin JM, Yang L, Artieri CG, van Baren, M J, Boley N, Booth BW, Brown JB, Cherbas L, Davis Ca, Dobin A, Li R, Lin W, Malone JH, Mattiuzzo NR, Miller D, Sturgill D, Tuch BB, Zaleski C, Zhang D, Blanchette M, Dudoit S, Eads B, Green RE, Hammonds $A$, Jiang L, Kapranov $P$, et al: The developmental transcriptome of Drosophila melanogaster. Nature 2011, 471(7339):473-479.

14. Parchman TL, Geist KS, Grahnen Ja, Benkman CW, Buerkle CA: Transcriptome sequencing in an ecologically important tree species: assembly, annotation, and marker discovery. BMC Genomic 2010, 11:180.

15. Wang Z, Fang B, Chen J, Zhang X, Luo Z, Huang L, Chen X, Li Y: De novo assembly and characterization of root transcriptome using Illumina paired-end sequencing and development of cSSR markers in sweet potato (Ipomoea batatas). BMC Genomics 2010, 11:726.

16. Der JP, Barker MS, Wickett NJ, DePamphilis CW, Wolf PG: De novo characterization of the gametophyte transcriptome in bracken fern, Pteridium aquilinum. BMC Genomics 2011, 12:99.

17. Garg R, Patel RK, Jhanwar S, Priya P, Bhattacharjee A, Yadav G, Bhatia S, Chattopadhyay D, Tyagi AK, Jain M: Gene discovery and tissue-specific transcriptome analysis in chickpea with massively parallel pyrosequencing and web resource development. Plant Physio/ 2011 156(August):1661-1678

18. Garg R, Patel RK, Tyagi AK, Jain M: De novo assembly of chickpea transcriptome using short reads for gene discovery and marker identification. DNA Res 2011, 18:53-63.

19. Hao DC, Ge G, Xiao P, Zhang Y, Yang L: The first insight into the tissue specific taxus transcriptome via Illumina second generation sequencing. Plos One 2011, 6(6):e21220.

20. Fernandez D, Tisserant E, Talhinhas P, Azinheira H, Vieira A, Petitot As, Loureiro A, Poulain J, DA Silva C, Silva MDC, Duplessis S:

454-pyrosequencing of Coffea arabica leaves infected by the rust fungus Hemileia vastatrix reveals in planta-expressed pathogen-secreted proteins and plant functions in a late compatible plant-rust interaction. Mol Plant Pathol 2012, 13:17-37.

21. Ralph SG, Chun HJE, Kolosova N, Cooper D, Oddy C, Ritland CE, Kirkpatrick R, Moore R, Barber S, Holt Ra, Jones SJM, Marra Ma, Douglas CJ, Ritland K, Bohlmann J: A conifer genomics resource of 200,000 spruce (Picea spp.) ESTs and 6,464 high-quality, sequence-finished full-length cDNAs for Sitka spruce (Picea sitchensis). BMC Genomics 2008, 9:484

22. Rigault P, Boyle B, Lepage P, Cooke JEK, Bousquet J, Mackay JJ: A white spruce gene catalog for conifer genome analyses. Plant Physiol 2011 157:14-28.

23. Lamothe $M$, Meirmans $P$, Isabel N: A set of polymorphic EST-derived markers for Picea species. Mol Ecol Notes 2006, 6:237-240.

24. Ralph SG, Yueh H, Friedmann M, Aeschilman D, Zeznik JA, Nelson CC Butterfield YSN, Kirkpatrick R, Liu J, Jones SJM, Marra MA, Douglas CJ Ritland K, Bohlmann J: Conifer defence against insects: microarray gene expression profiling of Sitka spruce (Picea sitchensis) induced by mechanical wounding or feeding by spruce budworms (Choristoneura occidentalis) or white pine weevils (Pissodes strobi) reveals large-scale. Plant, Cell Environ 2006, 29(8):1545-1570.

25. Liewlaksaneeyanawin C, Zhuang J, Tang M, Farzaneh N, Lueng G, Cullis C, Findlay S, Ritland CE, Bohlmann J, Ritland K: Identification of COS markers in the Pinaceae. Tree Genet Genomes 2008, 5:247-255.

26. Buschiazzo E, Ritland C, Bohlmann J, Ritland K: Slow but not low: genomic comparisons reveals slower evolutionary rate and higher dN/dS in conifers compared to angiosperms. BMC Evol Biol 2012, 12:8.

27. Renner S: Gymnosperms. In The time tree of Life. Edited by Hedges SB, Kumar S. New York: Oxford University Press; 2009.

28. Jaramillo-Correa JP, Verdú M, González-Martínez SC: The contribution of recombination to heterozygosity differs among plant evolutionary lineages and life-forms. BMC Evol Biol 2010, 10:22.

29. Moritsuka E, Hisataka Y, Tamura M, Uchiyama K, Watanabe A, Tsumura Y, Tachida $\mathrm{H}$ : Extended linkage disequilibrium in noncoding regions in a conifer, cryptomeria japonica. Genetics 2012, 190(3):1145-1148.

30. Ahuja MR, Neale DB: Evolution of genome size in conifers. Silvae Genetica 2005, 3(54):126-137.

31. Morgante M, De Paoli E: Genetics, Genomics and Breeding of Conifers, Chapter: Toward the conifer genome sequence. Genetics, Genomics and
Breeding of Crops Plants. New York: Edenbridge Science Publisher \& CRC Press; 2011

32. Evert RF, Raven PH, Eichorn SE: Biology of Plants. New York, USA: W.H.Freeman \& Co Ltd; 2005

33. Chen J, Källman T, Gyllenstrand N, Lascoux M: New insights on the speciation history and nucleotide diversity of three boreal spruce species and a Tertiary relict. Heredity 2010, 104:3-14.

34. Gernandt DS, Magallón S, López GG, Flores OZ, Journal I, Lo GG: Use of simultaneous analysis to guide fossil-based calibrations of Pinaceae phylogeny. Int J Plant Sci 2008, 169(8):1086-1099.

35. Zerbino DR, Birney E: Velvet: algorithms for de novo short read assembly using de Bruijn graphs. Genome Res 2008, 18(5):821-829.

36. Simpson JT, Wong K, Jackman SD, Schein JE, Jones SJM, Birol I: ABySS: a parallel assembler for short read sequence data. Genome Res 2009, 19(6):1117-1123.

37. Duvick J, Fu A, Muppirala U, Sabharwal M, Wilkerson MD, Lawrence CJ, Lushbough C, Brendel V: PlantGDB: a resource for comparative plant genomics. Nucleic Acids Res 2008, 36(Database issue):D959-D965.

38. Ran JH, Wei XX, Wang XQ: Molecular phylogeny and biogeography of Picea (Pinaceae): implications for phylogeographical studies using cytoplasmic haplotypes. Mol Phylogenet Evol 2006, 41(2):405-419.

39. Li Y, Stocks M, Hemmilä S, Källman T, Zhu H, Zhou Y, Chen J, Liu J, Lascoux M: Demographic histories of four spruce (Picea) species of the Qinghai-Tibetan Plateau and neighboring areas inferred from multiple nuclear loci. Mol Biol Evol 2010, 27(5):1001-1014.

40. Bouillé $M$, Senneville S, Bousquet J: Discordant mtDNA and cpDNA phylogenies indicate geographic speciation and reticulation as driving factors for the diversification of the genus Picea. Tree Genet Genomes 2010, 7(3):469-484

41. Schulz MH, Zerbino DR, Vingron M, Birney E: Oases: Robust de novo RNA-seq assembly across the dynamic range of expression levels. Bioinformatics 2012:1-7.

42. Wang Z, Gerstein M, Snyder M: RNA-Seq: a revolutionary tool for transcriptomics. Nat Rev Genet 2009, 10:57-63.

43. Kirst M, Johnson AF, Baucom C, Ulrich E, Hubbard K, Staggs R, Paule C, Retzel E, Whetten R, Sederoff R: Apparent homology of expressed genes from wood-forming tissues of loblolly pine (Pinus taeda L.) with Arabidopsis thaliana. Proc Nat Acad Sci U S A 2003, 100(12):7383-7388

44. Pavy N, Paule C, Parsons L, Crow Ja, Morency MJ, Cooke J, Johnson JE, Noumen E, Guillet-Claude C, Butterfield Y, Barber S, Yang G, Liu J, Stott J, Kirkpatrick R, Siddiqui A, Holt R, Marra M, Seguin A, Retzel E, Bousquet J, Mackay J: Generation, annotation, analysis and database integration of 16,500 white spruce EST clusters. BMC Genomics 2005, 6:144

45. Murray B, LI J, MD B: Gymnosperm DNA C-values database (release 4.0). 2010

46. Gan X, Stegle O, Behr J, Steffen JG, Drewe P, Hildebrand KL, Lyngsoe R, Schultheiss SJ, Osborne EJ, Sreedharan VT, Kahles A, Bohnert R, Jean G, Derwent P, Kersey P, Belfield EJ, Harberd NP, Kemen E, Toomajian C, Kover PX, Clark RM, Rätsch G, Mott R: Multiple reference genomes and transcriptomes for Arabidopsis thaliana. Nature 2011, 477(7365):419-423.

47. Heuertz M, De Paoli E, Källman T, Larsson H, Jurman I, Morgante M, Lascoux M, Gyllenstrand N: Multilocus patterns of nucleotide diversity, linkage disequilibrium and demographic history of Norway spruce [Picea abies (L.) Karst]. Genetics 2006, 174(4):2095-2105.

48. Kleinman CL, Majewski J: Comment on "Widespread RNA and DNA sequence differences in the human transcriptome". Science 2012, 335(6074):1302. author reply 1302

49. Lin W, Piskol R, Tan MH, Li JB: Comment on "Widespread RNA and DNA sequence differences in the human transcriptome". Science 2012 , 335(6074):1302. author reply 1302.

50. Pickrell JK, Gilad Y, Pritchard JK: Comment on “Widespread RNA and DNA sequence differences in the human transcriptome". Science 2012, 335(6074):1302. author reply 1302.

51. Willyard A, Ann W, Syring J, Gernandt DS, Liston A, Cronn R: Fossi calibration of molecular divergence infers a moderate mutation rate and recent radiations for pinus. Mol Biol Evol 2007, 24:90-101.

52. Smith Sa, Donoghue MJ: Rates of molecular evolution are linked to life history in flowering plants. Science 2008, 322(5898):86-89. 
53. Berlin $S$, Lagercrantz $U$, von Arnold S, Ost T, Rönnberg-Wästljung AC: High-density linkage mapping and evolution of paralogs and orthologs in Salix and Populus. BMC Genomics 2010, 11:129.

54. Yang $L$, Gaut $B S$ : Factors that contribute to variation in evolutionary rate among Arabidopsis genes. Mol Biol Evol 2011, 28(8):2359-2369.

55. Ingvarsson PK: Gene expression and protein length influence codon usage and rates of sequence evolution in Populus tremula. Mol BiOl Evol 2007, 24(3):836-844.

56. Wolf JBW, Künstner A, Nam K, Jakobsson M, Ellegren H: Nonlinear dynamics of nonsynonymous ( $d N$ ) and synonymous (dS) substitution rates affects inference of selection. Genome Biol Evol 2009, 1:308-319.

57. Ratnakumar A, Mousset S, Glémin S, Berglund J, Galtier N, Duret L, Webster MT: Detecting positive selection within genomes: the problem of biased gene conversion. Philos Trans R Soc London Ser B Biol sci 2010, 365(1552):2571-2580.

58. Stoletzki N, Eyre-Walker A: The positive correlation between $\mathbf{d N} / \mathbf{d S}$ and dS in mammals is due to runs of adjacent substitutions. $\mathrm{Mol} \mathrm{BiO} /$ Evol 2011, 28(4):1371-1380.

59. Subramanian S, Kumar S: Gene expression intensity shapes evolutionary rates of the proteins encoded by the vertebrate genome. Genetics 2004, 168:373-381.

60. Pál C, Papp B, Hurst LD: Highly expressed genes in Yeast evolve slowly. Genetics 2001, 158:927-931.

61. Slotte T, Bataillon T, Hansen TT, Onge KS, Wright SI, Schierup MH: Genomic determinants of protein evolution and polymorphism in arabidopsis. Mol Biol 2011, 3:1210-1219.

62. Jiao $Y, M a L$, Strickland E, Deng XW: Conservation and divergence of light-regulated genome expression patterns during seedling development in rice and Arabidopsis. Plant cell 2005 17(12):3239-3256.

63. Ma L, Sun N, Liu X, Jiao Y, Zhao H, Deng XW: Organ-specific expression of Arabidopsis genome during development. Plant Physiol 2005, 138:80-91.

64. Filichkin Sa, Breton G, Priest HD, Dharmawardhana P, Jaiswal P, Fox SE, Michael TP, Chory J, Kay Sa, Mockler TC: Global profiling of rice and poplar transcriptomes highlights key conserved circadian-controlled pathways and cis-regulatory modules. PloS one 2011, 6(6):e16907.

65. Alosi MC, Neale DB, Kinlaw CS: Expression of cab genes in Douglas-Fir is not strongly regulated by light. Plant Physio/ 1990, 93(2):829-832.

66. Gustafsson P, Jansson S, Lidholm J, Lundberg Ak: Structure and regulation of photosynthesis genes in Pinus sylvestris (Scots pine) and Pinus contorta (lodgepole pine). Forest Ecol Manage 1991, 43:287-300.

67. Cahais V, Gayral P, Tsagkogeorga G, Melo-Ferreira J, Ballenghien M, Weinert L, Chiari Y, Belkhir K, Ranwez V, Galtier N: Reference-free transcriptome assembly in non-model animals from next-generation sequencing data. Mol Ecol Resour 2012:834-845.

68. Norway spruce development of buds. Vienna: S2.02.11, International Union of Forest Research Organization; 1973.

69. Kolosova N, Miller B, Ralph S, Ellis BE, Douglas C, Ritland K, Bohlmann J: Isolation of high-quality RNA from gymnosperm and angiosperm trees. BioTechniques 2004, 36(5):821-824.

70. Fox S, Filichkin S, Mockler TC: Plant systems biology. Analysis 2009 553:79-108.

71. Feldmeyer B, Wheat CW, Krezdorn N, Rotter B, Pfenninger M: Short read Illumina data for the de novo assembly of a non-model snail species transcriptome (Radix balthica, Basommatophora, Pulmonata), and a comparison of assembler performance. BMC Genomics 2011, 12:317.

72. Pertea G, Huang X, Liang F, Antonescu V, Sultana R, Karamycheva S, Lee $Y$, White J, Cheung F, Parvizi B, Tsai J, Quackenbush J: TIGR Gene Indices clustering tools (TGICL): a software system for fast clustering of large EST datasets. Bioinformatics 2003, 19(5):651-652.

73. Li H, Durbin R: Fast and accurate short read alignment with Burrows-Wheeler transform. Bioinformatics 2009, 25(14):1754-1760.

74. Hardcastle TJ, Kelly Ka: baySeq: empirical Bayesian methods for identifying differential expression in sequence count data. $B M C$ Bioinf 2010, 11:422.
75. Li H, Handsaker B, Wysoker A, Fennell T, Ruan J, Homer N, Marth G, Abecasis G, Durbin R: The Sequence Alignment/Map format and SAMtools. Bioinformatics 2009, 25(16):2078-2079.

76. Liu L, Pearl DK: Species trees from gene trees: reconstructing Bayesian posterior distributions of a species phylogeny using estimated gene tree distributions. Syst Bio/ 2007, 56(3):504-514.

77. Rice P, Longden I, Bleasby A: EMBOSS: the European molecular biology open software suite. Trends Genet : TIG 2000, 16(6):276-277.

78. Lassmann T, Sonnhammer ELL: Kalign-an accurate and fast multiple sequence alignment algorithm. BMC Bioinf 2005, 6:298.

79. Wernersson R: RevTrans: multiple alignment of coding DNA from aligned amino acid sequences. Nucleic Acids Res 2003, 31(13):3537-3539.

80. Yang Z: PAML 4: phylogenetic analysis by maximum likelihood. $\mathrm{Mo}$ Biol Evol 2007, 24(8):1586-1591.

doi:10.1186/1471-2164-13-589

Cite this article as: Chen et al:: Sequencing of the needle transcriptome from Norway spruce (Picea abies Karst L.) reveals lower substitution rates, but similar selective constraints in gymnosperms and angiosperms. BMC Genomics 2012 13:589.

\section{Submit your next manuscript to BioMed Central and take full advantage of:}

- Convenient online submission

- Thorough peer review

- No space constraints or color figure charges

- Immediate publication on acceptance

- Inclusion in PubMed, CAS, Scopus and Google Scholar

- Research which is freely available for redistribution 